Voix et Images

volxetimages

\title{
Bibliographie Gilles Marcotte 1955-1979
}

\section{Louise Pelletier}

Volume 6, numéro 1, automne 1980

Gilles Marcotte

URI : https://id.erudit.org/iderudit/200248ar

DOI : https://doi.org/10.7202/200248ar

Aller au sommaire du numéro

Éditeur(s)

Les Presses de l'Université du Québec

ISSN

0318-9201 (imprimé)

1705-933X (numérique)

Découvrir la revue

Citer ce document

Pelletier, L. (1980). Bibliographie Gilles Marcotte 1955-1979. Voix et Images, 6(1), 35-49. https://doi.org/10.7202/200248ar d'utilisation que vous pouvez consulter en ligne.

https://apropos.erudit.org/fr/usagers/politique-dutilisation/ 


\title{
Bibliographie Gilles Marcotte 1955-1979
}

\author{
par Louise Pelletier
}

Comme son titre l'indique, cette bibliographie couvre 25 ans de production littéraire d'un des critiques les plus prolifiques de chez-nous: Gilles Marcotte. Cette limitation dans le temps a été rendue nécessaire par l'ampleur de l'œuvre critique de Gilles Marcotte. Le chercheur qui désirerait couvrir la période antérieure à 1955 trouvera dans le fichier Hamel un excellent outil de travail; on peut consulter ce fichier à la bibliothèque des sciences humaines et sociales de l'Université de Montréal.

La présente bibliographie compte près de 300 titres classés par ordre chronologique sous quatre rubriques: romans, critique littéraire, préfaces, textes pour la radio et la télévision. La seconde rubrique, de loin la plus importante, regroupe aussi bien les articles de journaux que les essais dont Gilles Marcotte est l'auteur. II faut souligner enfin que cette bibliographie, qui se veut exhaustive pour la période couverte, ne relève que les textes écrits par Gilles Marcotte et exclut ainsi toute critique ou compte rendu sur l'œuvre même de Marcotte.

\section{I) Romans}

Le poids de Dieu; roman. Paris, Flammarion, 1962. $218 p$.

El Peso de Dios. Traduction espagnole de Jesus Lopez Pacheco. (titre original : Le poids de Dieu). Barcelona, Luis de Caralt, 1963. 209 p.

The Burden of God. Traduction anglaise d'Elizabeth Abbott. (titre original : Le poids de Dieu). New York, Vanguard Press, 1964. $185 \mathrm{p}$.

Retour à Coolbrook; roman. Paris, Flammarion, 1965. 220 p.

Un voyage; récit. Montréal, $\mathrm{HMH}, 1973.185$ p. (Coll. l'Arbre). 


\section{2) Critique littéraire}

\section{Ouvrages}

Une littérature qui se fait; essais critiques sur la littérature canadiennefrançaise. Montréal, HMH, 1962. 293 p.

- Édition augmentée en 1968.

L'aventure romanesque de Claude Jasmin : Montréal, Département d'études françaises (Univ. de Montréal), 1965. $28 \mathrm{p}$.

Présence de la critique; critique et littérature contemporaines au Canada français. Textes choisis par Gilles Marcotte. Montréal, HMH, 1966. $254 \mathrm{p}$.

Description critique de la "poésie nouvelle" au Canada français. Thèse Univ. Laval (Québec). 1968.

Le temps des poètes; description critique de la poésie actuelle au Canada français. Montréal, $\mathrm{HMH}, 1969.247 \mathrm{p}$.

Les bonnes rencontres; chroniques littéraires. Montréal, HMH, 1971. 224 p. (Coll. Reconnaissances).

Le roman à l'imparfait; essais sur le roman québécois d'aujourd'hui. Montréal, La Presse, 1976. 194 p. (Coll. Échanges).

Anthologie de la littérature québécoise sous la direction de Gilles Marcotte. 4 vol. Montréal, La Presse, 1978.

\section{Articles}

«Moi, mes souliers». Le Devoir, 2 avril 1955, p. 6, col. 1 et 2.

«Sur la brèche». Le Devoir, 2 avril 1955, p. 6, col. 3 et 4.

«Un trésor dans la montagne». Le Devoir, 2 avril 1955, p. 6, col. 4 et 5 .

«Description de la culture de l'lle verte». Le Devoir, 2 avril 1955, p. 6, col. 5 et 6.

«Saint-Denys-Garneau (étude)». Les Écrits du Canada français, vol. III, 1957, p. 135-231.

«Un chrétien nommé Mounier». Cité Libre, fév. 1957, n 16, p. 3-8.

«Trois personnages ". Cité Libre, nov. 1957, n 18, p. 46-50.

“Alain Grandbois". Cité Libre, mai 1958, p. 33-35.

"Poésie canadienne française: une poésie d'exil". Mercure de France, tome 333, mai-août 1958, p. 5-9.

«Notes sur la jeune poésie». Cité Libre, juil. $58, n^{\circ} 21$, p. 39-42.

"La critique sociale dans le cinéma américain". Trois aspects du cinéma américain, Le Centre diocésain du cinéma de Montréal, 1959, p. 13-22.

«Recherche des signes». Liberté, janv.-fév. 1959, n 1, p. 45-49.

«Roman et journal intime». Liberté, mars-avril 1959, $n^{\circ} 2$, p. 104-107. 
«Deux vétérans: Desrosiers et Giroux». Liberté, mai-juin 1959, vol. 1, $n^{\circ} 3$, p. 181-186.

“Deux conseils". Liberté, juil.-août 1959, n 4, p. 256-258.

“Une poésie d'exil ». Canadian Literature, 2 (Autumn 1959), p. 32-36.

"Les Écrits du Canada français". Le Devoir, 19 mars 1960, p. 11, col. 1 à 5. "Le premier roman de Viviane de Silva : Visage de fièvre". Le Devoir, 2 avril 1960, p. 11, col. 3 à 7.

«M. André Chamson prévoit un rapprochement des communautés linguistiques ». Le Devoir, 5 avril 1960, p. 7, col. 7-8.

«Paul Toupin, prosateur: Souvenirs pour demain». Le Devoir, 16 avril 1960 , p. 10 , col. 5 à 8.

"Deux romans inachevés". Le Devoir, 30 avril 1960, p. 11, col. 6-7.

«Une romancière de vingt ans". Canadian Literature, 3 (Winter 1960), p. 71-72.

«Roman français 1961 ». Liberté, mars-avril 1961, vol. 3, n², p. 496-498.

«Critique universitaire». Liberté, mai-août 1961, vol. 3, $n^{\circ}$ 15-16, p. 648-651.

«L'Église et la culture». Informations catholiques internationales, Paris, $n^{\circ} 170,15$ juin 1962, p. 3-4.

"L'expérience du vertige dans le roman canadien-français (Essai) ". Écrits du Canada trançais, vol. XVI, 1963, p. 229-246.

"La religion dans la littérature canadienne-française contemporaine". Littérature et société canadiennes-françaises, publié sous la direction de Fernand Dumont et Jean-Charles Falardeau. Québec, P.U.L., 1964, p. 167-176.

«Témoignages des romanciers canadiens-français : Gilles Marcotte ». Archives des lettres canadiennes, vol. 3, 1964, p. 369-371.

"La religion dans la littérature canadienne-française". Recherches sociographiques, vol. 5, nos 1-2 (Québec, 1964), p. 167-176.

«Arts et lettres : Diplomate, essayiste et poète, Pierre Trottier, nouvel attaché culturel du Canada à Paris". Québec 64, vol. 1, n² 2, octobre 1964, p. 92-95.

«Revue des livres: Ethel et le terroriste de Claude Jasmin; Quelqu'un pour m'écouter de Réal Benoît». Québec 64, vol. 1, n², octobre 1964, p. 120-122.

"Refusant le prix Nobel de littérature, Sartre a-t-il raison ou pas?". Le Devoir, 31 octobre 1964, p. 11, col. 6.

«Les critiques littéraires de Montréal proposent leurs choix de livres pour Noël ». Le Devoir, 19 décembre 1964, p. 13, col. 6-7.

"Jean Basile et le goût de parler ". La Presse, 9 janvier 1965, supplément Arts et Lettres, p. 7, col. 3-4.

“Prix du Cercle: Georges Cartier ». La Presse, 9 janvier 1965, supplément Arts et lettres, p. 7, col. 1-2. 
“Prix France-Canada: J.-P. Pinsonneault». La Presse, 9 janvier 1965, supplément Arts et lettres, p. 6, col. 2-3.

“Réflexion sur une année littéraire de qualité ». La Presse, 9 janvier 1965, supplément Arts et lettres, p. 6, col. 1.

“Contes et nouvelles d'ici : Jacques Ferron, Jean Simard, Roch Carrier ". La Presse, 16 janvier 1965, supplément Arts et lettres, p. 6, col. 1-4.

“Quand les prix littéraires se multiplient...". La Presse, 16 janvier 1965, supplément Arts et Lettres, p. 1, col. 1-6.

“Littérature». La Presse, 23 janvier 1965, supplément Arts et lettres, p. 6, col. 1-4.

“Des professeurs qui écrivent ». La Presse, 30 janvier 1965, supplément Arts et lettres, p. 2, col. 4.

"Les livres canadiens-français sous la loupe de quelques sociologues et écrivains". La Presse, 30 janvier 1965, supplément Arts et lettres, p. 8, col. 1-2.

“La réalité, l'imagination, les écrivains...". La Presse, 30 janvier 1965, supplément Arts et lettres, p. 6, col. 1-4.

“L'Édition du Tricentenaire des Oeuvres de Blaise Pascal». La Presse, 6 février 1965, supplément Arts et lettres, p. 7, col. 1-4.

«Fernand Ouellette : un langage accompli». La Presse, 6 février 1965, supplément Arts et lettres, p. 6, col. 1-4.

“Les îles de l'enfance, les Amériques de l'avenir". La Presse, 13 février 1965, supplément Arts et lettres, p. 6, col. 1-4.

“André Major : littérature médinnequébec ». La Presse, 6 mars 1965, supplément Arts et lettres, p. 6, col. 3-4.

“Andrée Maillet et les remparts de Québec ". La Presse, 6 mars 1965, Supplément Arts et lettres, p. 6, col. 1-2.

«Paul-Marie Lapointe: Le poème-jazz». La Presse, 13 mars 1965, supplément Arts et lettres, p. 6, col. 2-4.

“Diane Giguère et la légende intérieure ". La Presse, 20 mars 1965, supplément Arts et lettres, p. 6, col. 1-4.

"Jacques Godbout: a mari usque ad mare». La Presse, 27 mars 1965, supplément Arts et lettres, p. 6, col. 1-4.

“Montréal et ses romanciers». La Presse, 3 avril 1965, supplément Arts et lettres, p. 1.

“Gérard Bessette à l'école du nouveau roman». La Presse, 10 avril 1965, supplément Arts et lettres, p. 4, col. 1-4.

“Contes du jour, de la nuit, et du demi-jour... ». La Presse, 17 avril 1965, supplément Arts et lettres, p. 4, col. 1-6.

«Présence de Sylvain Garneau ». La Presse, 17 avril 1965, supplément Arts et lettres, p. 1, col. 1-6, p. 5, col. 1-3. 
"Poésie des deux rives ». La Presse, 24 avril 1965, supplément Arts et lettres, p. 4, col. 1-4.

"Réal Benoît: “...Je veux aller beaucoup plus loin".. La Presse, $1^{\text {er }}$ mai 1965, supplément Arts et lettres, p. 1, col. 1-5, p. 4, col. 1-6.

“Parlez-nous d'amour». La Presse, 8 mai 1965, supplément Arts et lettres, p. 4, col. 1-4.

“Arthur Buies, Oedipe et la lanterne». La Presse, 15 mai 1965, supplément Arts et lettres, p. 4, col. 1-4.

“Jean-Paul Desbiens, l'allégresse et l'inquiétude». La Presse, 22 mai 1965, supplément Arts et lettres, p. 5, col. 1-6.

"Retour à Haydn : Biggs et Rostopovich ». La Presse, 22 mai 1965, supplément Arts et lettres, 22 mai 1965, p. 9, col. 1-6.

“Un térnoignage romanesque sur L'Église du silence». La Presse, 22 mai 1965, supplément Arts et lettres, p. 4, col. 1-4.

“Jean-Paul Desbiens est un fier baveux". La Presse, 29 mai 1965, supplément Arts et lettres, p. 4, col. 1-4.

“Mlle Étincelle, détective en jupons». La Presse, 29 mai 1965, supplément Arts et lettres, p. 5, col. 1-3.

«Retour à Coolbrook (extrait)». Châtelaine, vol. 6, n6,6, juin 1965, p. 26, p. 65-72.

"La littérature qui se lit...". La Presse, 5 juin 1965, supplément Arts et lettres, p. 4, col. 1-4.

"Le Funambule de Lemoine". La Presse, 12 juin 1965, supplément Arts et lettres, p. 4, col. 1-4.

“Les Terres noires de Fugère ». La Presse, 12 juin 1965, supplément Arts et lettres, p. 4, col. 1-4.

«Lettre ouverte à Claude Jasmin, romancier». La Presse, 19 juin 1965, supplément Arts et lettres, p. 6, col. 1-4.

«Pour lire Dante». La Presse, 19 juin 1965, supplément Arts et lettres, p. 4, col. 1-6.

«L'Amour impossible». La Presse, 26 juin 1965, supplément Arts et lettres, p. 4, col. 1-4.

"En livre de poche, les romans vécus et les autres ". La Presse, 3 juil. 1965, supplément Arts et lettres, p. 5, col. 3-6.

"Quatre écrivains devant la question religieuse: Saint-Exupéry, Camus, Châteaubriand, Montaigne". La Presse, 3 juil. 1965, supplément Arts et lettres, p. 4, col. 1-4.

“Aragon l'enchanteur». La Presse, 10 juil. 1965, supplément Arts et lettres, p. 4, col. 1-4.

“La Slovénie est-elle plus près de nous que les États-Unis?». La Presse, 31 juil. 1965, p. 6, col. 1-4. 
«Paul Evdokimov et la tradition orientale». La Presse, 7 août 1965, supplément Arts et lettres, p. 5, col. 1-4.

"Poésie française : Haïti, Belgique, France». La Presse, 7 août 1965, supplément Arts et lettres, p. 4, col. 1-6.

«Un immortel de nos lettres: Jules Fournier». La Presse, 14 août 1965 , supplément Arts et lettres, p. 4, col. 1-4.

“De Russie et d'Amérique». La Presse, 4 sept. 1965, supplément Arts et lettres, p. 4, col. 1-4.

«Témoignages». Le Soleil, 11 sept. 1965, p. 28, col. 4.

«Et si nous relisions André Breton ?... ». La Presse, 18 sept. 1965, supplément Arts et lettres, p. 4, col. 1-4.

«Nous irons au théâtre avec Radio-Canada». La Presse, 25 sept. 1965, supplément Arts et lettres, p. 6, col. 1-6.

«Une Québécoise en Europe «rouge»". La Presse, 25 sept. 1965, supplément Arts et lettres, p. 5, col. 3-4.

«Georges Simenon, une moitié de chef-d'œuvre». La Presse, 2 oct. 1965, p. 4 .

"Une information pour les grandes et les petites circonstances ". La Presse, 2 oct. 1965, p. 10.

«Francis Ponge : redonner au langage sa fonction propre». La Presse, 9 oct. 1965, supplément Arts et lettres, p. 5, col. 1-6.

«Le premier Cahier du Cercle juif de langue française». La Presse, 9 oct. 1965, supplément Arts et lettres, p. 4, col. 1-6.

«Poésie et symbole de Paul Wyczynski ». La Presse, 16 oct. 1965, p. 4, col. 1-4.

«L'amour, aujourd'hui : Françoise Sagan et Suzy Morel ». La Presse, 23 oct. 1965, p. 4.

"Avez-vous reçu un prix? - Gérard Bessette». La Presse, 23 oct. 1965, p. 6.

“Avez-vous reçu un prix? - Jacques Brault». La Presse, 23 oct. 1965, p. 6. "Avez-vous reçu un prix ? - Roch Carrier ». La Presse, 23 oct. 1965, p. 6.

«Avez-vous reçu un prix ? - Paul-Marie Lapointe». La Presse, 23 oct. 1965, p. 6.

“Avez-vous reçu un prix? - Andrée Maillet". La Presse, 23 oct. 1965, p. 6. “Avez-vous reçu un prix? - Paul Toupin». La Presse, 23 oct. 1965, p. 6. «Le drame de l'instituteur laïque au Canada français». La Presse, 30 oct. 1965 , p. 4.

“Hubert Aquin et le destin d'écrivain ». La Presse, 6 nov. 1965, p. 3, col. 1-6. «Les infusoires de Monique Bosco». La Presse, 6 nov. 1965, p. 5, col. 1-6. “Alain Bosquet: I'absolu et la dérision ". La Presse, 6 nov. 1965, p. 4, col. 1-4. 
“Une bombe : Prochain épisode». La Presse, 13 nov. 1965, p. 4, col. 1-4.

“Brève rencontre avec Henri-Irénée Marrou ». La Presse, 13 nov. 1965, p. 6 , col. 1-5.

«Bertrand Vac : le scalpel et le stylo». La Presse, 20 nov. 1965, supplément Arts et lettres, p. 5.

"La grande voix de François Mauriac". La Presse, 20 nov. 1965, supplément Arts et lettres, p. 4.

"André Laurendeau, des Voyages au pays de l'enfance à Une Vie d'enfer". La Presse, 27 nov. 1965, supplément Arts et lettres, p. 1.

“De Montréal à Venise, l'échec du bonheur ». La Presse, 27 nov. 1965, supplément Arts et lettres, p. 4.

"Les grands thèmes du roman canadien : l'amour et l'érotisme". Liberté 42 , vol. $7, n^{\circ} 6$, nov-déc. 1965, p. 533.

"Les grands thèmes du roman canadien : le patriotisme". Liberté 42 , vol. 7 , $n^{\circ}$ 6, nov.-déc. 1965, p. 534.

“Les grands thèmes du roman canadien: la religion". Liberté 42, vol. 7 , $n^{\circ} 6$, nov.-déc. 1965, p. 535.

“Les grands thèmes du roman canadien: la vie». Liberté 42 , vol. $7, n^{\circ} 6$, nov.-déc. 1965, p. 535.

"Les grands thèmes du roman canadien: la mort». Liberté 42 , vol. $7, n^{\circ} 6$, nov.-déc. 1965, p. 536.

"Les grands thèmes du roman canadien : la femme". Liberté 42 , vol. $7, n^{\circ} 6$, nov.-déc. 1965, p. 537.

“De Roland Barthes à Françoise Sagan». La Presse, 4 déc. 1965, supplément Arts et lettres, p. 6, col. 1-3.

"Que diable allait-il faire dans cette'galère? ". La Presse, 4 déc. 1965, supplément Arts et lettres, p. 5, col. 4-6.

"Seize questions sur le dernier roman de Robbe-Grillet ". La Presse, 4 déc. 1965, supplément Arts et lettres, p. 4.

"Claire Martin, le vieux démon ironique et les surprises de la mémoire". La Presse, 11 déc. 1965, supplément Arts et lettres, p. 5.

“Deux langages ". La Presse, 11 déc. 1965, supplément Arts et lettres, p. 4. «Histoires galantes». La Presse, 18 déc. 1965, supplément Arts et lettres, p. 4.

"Une vie d'enfer". La Presse, 18 déc. 1965, supplément Arts et lettres, p. 4.

«Et puis tout est silence...». La Presse, 18 déc. 1965, supplément Arts et lettres, p. 4.

«Roland Giguère à l'âge de la parole». La Presse, 18 déc. 1965, supplément Arts et lettres, p. 1, 3.

“Dans un gant de fer ou l'histoire d'un combat". La Presse, 24 déc. 1965, supplément Arts et lettres, p. 4, col. 1-4. 
«Une introduction à l'œuvre d'Anne Hébert». La Presse, 24 déc. 1965, supplément Arts et lettres, p. 5, col. 1-2.

«Une histoire des années 60 : Les Choses de Georges Perec, prix Renaudot 1965 ». La Presse, 31 déc. 1965, supplément Arts et lettres, p. 4.

“Un soir de décembre, Hercule Savarin...». La Presse, 31 déc. 1965, supplément Arts et lettres, p. 4.

«Edgard Varèse de Fernand Ouellette». Livres et auteurs canadiens - 1966, p. 169-170.

"François Mauriac, les charmes d'une belle prose". Journal des Jeunesses Musicales du Canada, janvier 1966, p. 11, col. 1-2.

«Du neuf et du vieux (mais où le neuf, où le vieux?)". La Presse, 8 janv. 1966, supplément Arts et lettres, p. 4.

“Aux États-Unis, le plaisir de conter... ». La Presse, 15 janvier 1966, supplément Arts et lettres, p. 4.

«Andrée Maillet, Montréalaise». La Presse, 22 janv. 1966, supplément Arts et lettres, p. 5-A.

«Paul Toupin docteur en Berthelot Brunet». La Presse, 22 janvier 1966, supplément Arts et lettres, p. 4-A.

"Maintenant et L'Action Nationale: aggiornamento et séparatisme". La Presse, 29 janv. 1966, supplément Arts et lettres, p. 5, col. 1-6.

“Umberto Eco et l'œuvre ouverte». La Presse, 29 janv. 1966, supplément Arts et lettres, p. 4.

«Deux petits livres bien attachants". Journal des Jeunesses Musicales du Canada, février 1966, p. 11, col. 1-2.

“Nouvelles montréalaises». La Presse, 5 fév. 1966, supplément Arts et lettres, p. 4.

«François Nourissier, la France et la guerre». La Presse, 12 fév. 1966, supplément Arts et lettres, p. 4.

«Relations et Études». La Presse, 12 fév. 1966, supplément Arts et lettres, p. 6, col. 1-5.

“En finale: Minou Petrowski et Anne Bernard». La Presse, 19 fév. 1966, supplément Arts et lettres, p. 4, col. 1-4.

"Les humanités du XXe siècle: de Henri Van Lier à Gaston Bachelard". La Presse, 26 fév. 1966, supplément Arts et lettres, p. 4, col. 1-4.

“Cité libre, L'Action Nationale, Maintenant ". La Presse, 5 mars 1966, supplément Arts et lettres, p. 7, col. 1-4.

"Un livre de maître artisan : Deux cavaliers de l'orage de Giono". La Presse, 5 mars 1966, supplément Arts et lettres, p. 4, col. 1-4.

«Des nouvelles de Madeleine Ferron, des poèmes de Marcelle Desjardins et de François Piazza». La Presse, 12 mars 1966, supplément Arts et lettres, p. 4, col. 1-4. 
“Paul Claudel et l'Allemagne ». La Presse, 12 mars 1966, supplément Arts et lettres, p. 5, col. 5-6.

"Grandes éditions". La Presse, 19 mars 1966, supplément Arts et lettres, p. 5, col. 5-6.

«Les Revues". La Presse, 19 mars 1966, supplément Arts et lettres, p. 5, col. 1-4.

“Marguerite Duras et les gestes de la misère». La Presse, 19 mars 1966, supplément Arts et lettres, p. 4.

"Culture vivante : en rodage». La Presse, 26 mars 1966, supplément Arts et lettres, p. 5.

«Graham Greene et la comédie... inhumaine». La Presse, 26 mars 1966, supplément Arts et lettres, p. 4.

“La poule et l'œuf ». La Presse, 2 avril 1966, supplément Arts et lettres, p. 2. “L'arbre blanc, de Rina Lasnier». La Presse, 9 avril 1966, supplément Arts et lettres, p. 4.

"Lectures: hommage à Félix-Antoine Savard - La Revue de l'école normale: la philosophie en question". La Presse, 9 avril 1966, supplément Arts et lettres, p. 7.

«Parti pris s'intéresse au Crédit social - Esprit : prospective et utopie». La Presse, 16 avril 1966, supplément Arts et lettres, p. 5.

“Toutes les routes vont par Altamont...". La Presse, 16 avril 1966, supplément Arts et lettres, p. 4.

“Tchekhov à la russe». La Presse, 19 avril 1966, p. 36, col. 7-9.

"Livres et auteurs canadiens 1965 - Beaucoup d'auteurs et beaucoup de critiques...". La Presse, 23 avril 1966, supplément Arts et lettres, p. 5.

“Ouvrages de critique ». La Presse, 23 avril 1966, supplément Arts et lettres, p. 4.

"Jacques Ferron, grand-prêtre du vaudou québécois - Jacques Hébert et Les éc zurants ". La Presse, 30 avril 1966, supplément Arts et lettres, p. 4.

"Voulez-vous lire avec moâ?". La Presse, 30 avril 1966, supplément Arts et lettres, p. 5, col. 1-4.

«Culture: technique et humanisme-Dialogue: Faut-il psychanalyser Goethe?". La Presse, 7 mai 1966, supplément Arts et lettres, p. 5.

“Ernest Ansermet interprète Schumann, Mendelsohn et Debussy». La Presse, 7 mai 1966, supplément Arts et lettres, p. 9.

«Hugh MacLennan parmi nous ». La Presse, 7 mai 1966, supplément Arts et Lettres, p. 3.

«Oeuvres complètes : Francis Ponge et Pierre Jean Jouve». La Presse, 7 mai 1966, supplément Arts et lettres, p. 6, col. 5-6.

"Les Cahiers de Sainte-Marie: littérature canadienne - Culture vivante, pour quoi faire? - Les dix ans de Vie des arts ". La Presse, 14 mai 1966, supplément Arts et lettres, p. 7, col. 1-3. 
«Gilbert Choquette et I'apprentissage - Gilles Vigneault, conteur». La Presse, 14 mai 1966, supplément Arts et lettres, p. 4.

"Paul Robert: J'ai fait mon dictionnaire pour moi-même!". La Presse, 14 mai 1966, supplément Arts et lettres, p. 3.

"La famille, aujourd'hui : un numéro spécial des Cahiers de Droit de l'Université Laval ». La Presse, 21 mai 1966, supplément Arts et lettres, p. 5, col. 1-4.

«Suzanne Paradis: en chute libre..., - Les poèmes choisis de Frank R. Scott». La Presse, 21 mai 1966, supplément Arts et lettres, p. 4.

“Une nouvelle romancière, quelques poètes et de jeunes auteurs». La Presse, 28 mai 1966, supplément Arts et lettres, p. 4.

«Le premier roman de Hugh MacLennan». La Presse, 4 juin 1966, supplément Arts et lettres, p. 4.

"Socialisme 66 : dépendance et insécurité ". La Presse, 4 juin 1966, supplément Arts et lettres, p. 5, col. 1-4.

“Au fond, puisque c'est l'été, pourquoi ne pas...?". La Presse, 11 juin 1966, supplément Arts et lettres, p. 4.

“Maintenant: femme d'aujourd'hui et limitation des naissances - Cité libre : la coopérative, pour quoi faire? ". La Presse, 11 juin 1966, supplément Arts et lettres, p. 5, col. 1-4.

“Pour comprendre la littérature d'aujourd'hui». Journal des Jeunesses Musicales du Canada, septembre 1966, p. 10, col. 3-4, et p. 11, col. 1-2.

“Les poèmes de Robert Marteau ". Liberté, vol. 8, $\mathrm{n}^{\circ}$ 5-6, sept.-déc. 1966, p. 135-137.

“L'insoumise de Marie-Claire Blais". Québec 66, vol. 3, n 8, oct. 1966, p. 7981.

“Traduit du français". Lettres nouvelles (Paris), déc. 66-janv. 67, p. 78-84.

"Jean-Charles Falardeau, notre société et son roman ". Recherches sociographiques, vol. VIII, $n^{\circ} 2$ (1967), p. 227-230.

"Le Poids de Dieu". Québec: hier et aujourd'hui, Centre d'Études canadiennes-françaises, McGill University, Montreal, 1967, p. 30-32.

"Le romancier canadien-français et son juif ". Juifs et Canadiens (deuxième cahier du Cercle juif de langue française), Montréal, Ed. du Jour, 1967, p. 61-68.

«Difficulté de la poésie». Liberté, vol. 9, n 1, janv.-fév. 1967, p. 64-68.

"Dix ans de vie littéraire au Canada trançais de Pierre de Granpré ». Québec 67, vol. 4, février 1967, p. 103 et 104.

“Bilan de la course aux prix littéraires". Journal des jeunesses musicales du Canada, mars 1967, p. 10, col. 3-4.

«Fernand Ouellet, Histoire économique et sociale du Québec (1760-1850)". Études françaises, vol. 3, $n^{\circ} 2$, mai 1967, p. 234-239. 
«Godin, Garcia, Saint-Denys Garneau ». Liberté, vol. 9, n³, mai-juin 1967, p. 79-83.

"Les Cantouques de Gérald Godin», Québec 67, vol. 4, octobre 1967, p. 164-166.

"Situation de la littérature québécoise : française? oui ou non ». Le Devoir, 31 oct. 1967, p. VII, col. 1.

«D'une littérature nationale». Of Several branches : Essays from the Humanities Association Bulletin, Toronto, Univ. of Toronto Press, 1968, p. 170176.

“Jean Basile, le Grand Khan». Études françaises, vol. 4, n², mai 1968, p. 230-232.

“André Laurendeau ou les nécessités de la parole". Le Devoir, 8 juin 1968, p. 11 , col. 1.

«Le congé de René Char, (poème) ». Liberté, vol. 10, $n^{\circ} 4$, juillet-août 1968, p. 63-72.

“Table ronde sur la création artistique». (Exposés de Gilles Marcotte, Robert Frith, James Domville, Gilles Tremblay, Jean-René Ostiguy suivis d'une discussion) dans Le Canada au seuil du siècle de l'abondance ; entretiens de Cerisy-la-Salle 1968, Montréal, HMH, 1969, p. 282303.

"La critique des journaux et des revues» dans Pierre de Grandpré, Histoire de la littérature française du Québec, tome IV, Montréal, Beauchemin, 1969, p. 341-352.

"L'aventure romanesque de Claude Jasmin". Littérature canadienne-française; conférences J. A. de Sève. Montréal, P.U.M., 1969, p. 89-112.

"Jacques Poulin, Jimmy". Études françaises, vol. 5, n², mai 1969, p. 236237.

«Méchant Michaux». Liberté, vol. 11, n 6, nov.-déc. 1969, p. 19-21.

"La poésie". Études françaises, vol. 6, n², mai 1970, p. 227-241.

"Berthelot Brunet tel qu'en lui-même. Anthologie préparée par Gilles Marcotte". Écrits du Canada français, $n^{\circ} 29,1970$, p. 9-122.

"Le son et l'image; textes pour la radio et la télévision". Écrits du Canada français, $\mathbf{n}^{\circ} 30,1970$, p. 151-190.

"Notes sur le thème du pays". Voix et images du pays, IV, 1971, p. 11-26.

“La poésie". Études françaises, vol. VII, $n^{\circ} 1$, fév. 1971, p. 102-114.

"La recherche du pays dans la poésie canadienne-française ". Revue générale; perspectives européennes des sciences humaines, Bruxelles, vol. $107, n^{\circ} 6$, juin 1971, p. 13-26.

«Littérature canadienne de langue française». Dizionario critico della letterature francese (Simone Franco, ed). Turin, Unione Tipografico, 1972, p. 670-676.

“Qui a peu du pygargue roux?». Liberté, XIV, $n^{\circ}$ 6, 1972, p. 94-115. 
“Jean-Aubert Loranger». Écrits du Canada français, $n^{\circ} 35,1972, p .11-12$. “La poésie - pour l'âme». Études françaises, VIII, $n^{\circ}$ 1, fév. 1972, p. 87-100. “Naître à Sherbrooke». Le Devoir, 28 oct. 1972, p. XXVII, col. 1. “Les deux enfers de Rimbaud». Liberté, vol. XV, $n^{\circ} 314,1973, p .162-167$. "Robert Elie - pour l'amitié". Le Devoir, 27 janv. 1973, p. 17, col. 1. "La poésie, le poète et ses mots ". Études trançaises, vol. IX, n 1, fév. 1973, p. 74-89.

«Roland Barthes et la poêle à frire". Le Devoir, 24 mars 1973, p. 16, col. 2. "Jeu de roman, jeu de souterrain...". Le Devoir, 7 avril 1973, p. 20, col. 1. «Histoire de mer et de terre». Le Devoir, 21 avril 1973, p. 17, col. 1. “Saint-John Perse par lui-même». Le Devoir, 12 mai 1973, p. 18, col. 1 «De James en Giacomo». Le Devoir, 26 mai 1972, p. 15, col. 1. «J.M.G. Le Clézio et les Machines». Le Devoir, 9 juin 1973, p. 14, col. 1. «Jean Amrouche et Guiseppe Ungaretti, homme de peine». Le Devoir, 23 juin 1973, p. 14, col. 1.

“Images du monde actuel : récits de Mohammed Dib et Yves Navarre ". Le Devoir, 18 août 1973, p. 12, col. 1.

“Du côté de la critique: R. Jakobson et J. Derrida". Le Devoir, $1^{\text {er }}$ sept. 1973, p. 14, col. 1.

«Le bidjac de Lescure». Le Devoir, 22 sept. 1973, p. 17, col. 4.

“Qui est Marcel Fournier? », Le Devoir, 6 oct. 1973, p. 15, col. 1.

"L'Enseignement de la littérature - Les programmes: si Charles Baudelaire revenait...". Le Devoir, 10 nov. 73 , p. XIII, col. 1.

“Communication à la Rencontre québécoise internationale des écrivains sur le roman des Amériques". Liberté, vol. 15, no. 6, nov.-déc. 1973, p. 102-106.

"La littérature canadienne française d'aujourd'hui". Le français dans le monde, Paris, no. 101, déc. 1973, p. 6-10.

“Un roman de l'attention: Pentecôte». Le Devoir, 1er déc. 1973, p. 15, col. 1.

“Les mots comme des choses". Études françaises, vol. X, 1974, p. 125136.

"Présence de l'Absent: Ies mémoires de Jacques Madaule". Le Devoir, 16 fév. 1974, p. 18 , col. 1.

"Poètes d'hier et d'aujourd'hui: P. Reverdy et R. Bodart". Le Devoir, 2 mars 1974, p. 18, col. 1.

"Qu'est-ce qu'un grand écrivain? Giono, par exemple...". Le Devoir, 16 mars 1974, p. 19, col. 1.

"Peut-on parler du $H$ (de Sollers) dans un journal?". Le Devoir, 30 mars 1974, p. 18, col. 1. 
"Sèche précision et grâce légère". Le Devoir, 20 avril 1974, p. 16, col. 1.

"Lettres françaises: le dernier Malraux; l'histoire, l'art». Le Devoir, 4 mai 1974 , p. 15, col. 5.

“Michel de Certeau et la Culture au pluriel». Le Devoir, 18 mai 1974, p. 24 , col. 1.

"Trois partitions émouvantes de Marguerite Duras". Le Devoir, 1er juin 1974, p. 15, col. 1.

"Marshall McLuhan et l'énergie du banal». Le Devoir, 15 juin 1974, p. 17, col. 1.

“Marguerite Yourcenar: “L'être que j'appelle moi...". Le Devoir, 14 sept. 1974, p. 16, col. 1.

"D'un ancien et de quelques modernes (dont Baudelaire)". Le Devoir, 28 sept. 1974, p. 17 , col. 1.

“Jean-Guy Pilon, poète”. Liberté, vol. XVI, no. 5/6, sept.-déc. 1974, p. 54-55.

"La nouvelle poésie française à la Delvaille...". Le Devoir, 12 oct. 1974, p. 18 , col. 1.

"Encore et toujours". Le Devoir, 29 mars 1975, p. 15, col. 6.

«Réjean Ducharme contre Blasey Blasey». Études françaises, vol. XI, no. 3-4, oct. 1975, p. 247-284.

«Jacques Blais, Présence d'Alain Grandbois». Esprit créateur, hiver 1975, vol. $X V$, no. 4 , p. 472-473.

"Réjean Ducharme, Les Enfantômes". Livres et auteurs québécois (1976), p. 23-26.

“Pierre Pagé, Le comique et l'humour à la radio québécoise (1930-1970)". Livres et auteurs québécois (1976), p. 235-236.

«Jacques Ferron, côté village». Études françaises, vol. XII, oct. 1976, p. 217-236.

«Jacques Blais, De l’ordre et de l'aventure». Études littéraires, déc. 1976, vol. 9 , no. 3, p. 596-600.

"Alain et Abel". Langue, littérature, culture au Canada français (Robert Vigneault, ed.), Cahier du C.R.C.C.F., Ed. de I'Univ. d'Ottawa, 1977, p. 81-89.

"Moi, mon taureau, mon âme, la Grèce, etc...; Les cornes sacrées de Roger Fournier ". Le Devoir, 21 mai 1977, p. 28, col. 1.

“Les problèmes du capitaine». Liberté, vol. XIX, no. 3, mai-juin 1977, p. 7886.

"Une certaine idée de la tradition; Roch Carrier et le pays de grandpère». Le Devoir, 18 juin 1977, p. 19, col. 1.

«Fernand Ouellette, ici et ailleurs». Le Devoir, 9 juil, 1977, p. 12, col. 1.

«Où en sont les littératures nationales (Le Québec) ». Liberté, XIX, nos. 112113, juil.-oct. $1977,120-125$. 
"Andrée Maillet: quelques heures chez les riches". Le Devoir, 20 août 1977, p. 13, col. 1.

“Gabrielle Roy et l'institutrice passionnée». Le Devoir, 24 sept. 1977, p. 15, col. 1.

“Un lecteur nommé Alfred ". Le Devoir, 8 oct. 1977, p. 17, col. 1.

“Histoires de nous-mêmes; Madeleine Ferron, Jean-Paul Filion ". Le Devoir, 29 oct. 1977 , p. 29 , col. 1.

"L'Emmitouflé de Louis Caron". Le Devoir, 19 nov. 1977, p. 33, col. 1.

"Des chats et des hommes". Le Devoir, 10 déc. 1977, p. 36, col. 1.

«Nationalism \& literature in Quebec». Canadian Literature, no, 75, winter 1977, p. 6-14.

"Un amateur". Les Cahiers de l'Académie canadienne-française; vol. 15 Victor Barbeau, 1978, p. 122-128.

“La deuxième Saison en Enfer d'Arthur Rimbaud". Écrits du Canada français, vol. XLI, 1978, p. 167-191.

“À l'écoute du passé: Victor Barbeau, Paul Toupin». Le Devoir, 14 janv. 1978, p. 33, col. 1.

“La révolution de la tranquillité ". Revue de l'Université Laurentienne, (La poésie depuis 1950), vol. X, no. 2, fév. 1978, p. 67-75.

«Une saison dans la vie de Geneviève Aures». Le Devoir, 25 mars 1978, p. 35, col. 1.

“Des miroirs, pour quoi faire?». Liberté, vol. XX, no. 27, mars-avril 1978, p. 96-98.

“Gabrielle Roy: Chercheur d'horizon”. Le Devoir, 22 avril 1978, p. 29, col. 1. "Une saveur américaine". La Presse, 3 juin 1978, p. D2, col. 1.

"La Chine sans chinoiseries". Le Devoir, 23 juin 1978, p. 36, col. 6.

“Vampire \& chasseurs de vampires". La Presse, 30 juin 1978, p. C2, col. 5. "La haine manque de dents...". Le Devoir, 12 août 1978, p. 14, col. 1.

"Antoine Gérin-Lajoie, homme de lettres". Le Devoir, 9 sept. 1978, p. 23 , col. 1.

“Anne Hébert: prix David; les cinq «prix du Québec»1978». Le Devoir, 7 oct. 1978 , p. 19 , col. 1.

“La problématique du récit dans le roman québécois d'aujourd'hui ». Revue des sciences humaines (Lille III), no. 173, 1979-1, p. 59-69.

“Prendre tout pour une fenêtre". Le Devoir, 3 fév. 1979, p. 17, col. 1.

“Quand l'écrivain se fait personnage». Le Devoir, 17 mars 1979, p. 19. col. 1.

“Lisez Jacques Poulin, faites de beaux rêves!". Le Devoir, 12 mai 1979, p. 23, col. 1. 


\section{3) Préfaces}

The Journal of Saint-Denys-Garneau, translated by John Glassco. Toronto, McLelland \& Stewart, 1963.

Saint-Denys Garneau, Anne Hébert, Translations/Traductions by F.F. Scott. Vancouver, Klanak Press, 1964.

Sirois, Antoine. Montréal dans le roman canadien. Paris-Montréal, Didier, 1969.

Loranger, Jean-Aubert. Les Atmosphères suivi de Poèmes. Montréal, HMH, 1970 (Coll. Sur Parole).

Falardeau, Jean-Charles. Imaginaire social et littérature. Montréal, HMH, 1974. (Coll. Reconnaissances).

Maillet, Andrée. Profil de l'original. Montréal, L'Hexagone, 1974.

\section{4) Textes pour la radio et la télévision}

Au milieu de la course de notre vie... Trilogie dramatique présentée à la télévision de Radio-Canada en 1966. Réalisation de Jean-Paul Fugère.

La deuxième saison en enter d'Arthur Rimbaud. Série Documents. Émission de radio à CBF. 1976.

Schubert inachevé. Série Documents. Émission de radio à CBF. 1977.

Robert Musil et l'homme sans qualités. Série Documents. Émission de radio à CBF. 1978.

«Drame et musique: Alban Berg », série "Documents », Radio-Canada, 1978. 